Indian J Anim Health (2021), 60(2): 205-212

DOI: https://doi.org/10.36062/ijah.2021.08321

\title{
In silico analysis of bovine Glutathione-S-transferase M1 (GSTM1), an essential gene governing bull semen quality
}

\author{
A. Mukherjee ${ }^{1 *}$ I. Kar², S. Das ${ }^{3}$, A. P. Acharya ${ }^{1}$, H. K. Maity ${ }^{2}$, P. Biswas ${ }^{2}$ and S. Biswas ${ }^{2}$ \\ ${ }^{1}$ Department of Animal Biotechnology, West Bengal University of Animal and Fishery Sciences, \\ Mohanpur, Nadia - 741 252, West Bengal, India; ${ }^{2}$ Department of Avian Science, West Bengal \\ University of Animal and Fishery Sciences, Mohanpur, Nadia - 741 252, West Bengal, India; \\ ${ }^{3}$ Department of Fish Nutrition, West Bengal University of Animal and Fishery Sciences, Chakgaria, \\ Kolkata-700 094, West Bengal, India
}

\begin{abstract}
Artificial insemination has great applicability in the dairy industry globally. However, poor semen quality with subfertility in bulls is a major challenge to increase the conception rate following insemination. Glutathione-S-transferase M1 (GSTM1) is an important gene that regulates semen quality in a bull. The bovine GSTM1 protein sequence was retrieved; its three-dimensional structure was predicted and validated in silico in this study. The three-dimensional protein structure was predicted using Modeller 9.24, I-TASSER, Phyre 2 and Swiss Model. Structure validation was done by observing the Ramachandran Plot, Hydrophobicity Plot considering amino acid residues in the favored region and several other tools. The model predicted by Modeller 9.24 was the best model with $98.1 \%$ residues in the favored region, $0.5 \%$ in the allowed region and $1.4 \%$ in the outlier region. The predicted structure was docked with glutathione as ligand using PatchDock, and docked poses were analyzed using Ligplot plus. Interactomic analysis with STRING database reveals its interaction with other variants of Glutathione-S-transferase and cytochromeP450 family genes. Prediction of the structure of GSTM1 protein will improve our understanding of its function to determine bull semen quality. Also, the study will be a significant theoretical resource to conduct future studies aiming to develop candidate gene markers for selecting superior quality bulls.
\end{abstract}

Key words: Bull, Docking, Fertility, GSTM1, Three dimensional

\section{INTRODUCTION}

The widespread application of artificial insemination (AI) in dairy animals has a profound impact on the optimization of dairy productivity. The technique ensures rapid dissemination of superior genetic trait(s) while decreasing the economic burden of maintaining bulls. The genomic selection was introduced into dairy cattle development projects, with the goal of increasing rates of genetic gain, particularly for traits with low heritability like fertility and longevity (Garcia-Ruiz et al., 2016). Umpteen number of factors contributed by the male, female and/or management and/or environment may be the cause of sub/in-fertility in bulls. A thorough understanding of genomic, proteomic and metabolomic landscapes of spermatozoa through advanced in silico analysis and experimental validation is extremely required to elucidate the underlying mechanisms regulating sperm quality (Byrne et al., 2012; Jodar et al., 2015; Selvaraju et al., 2017).

Glutathione-S-transferases (GST; EC 2.5.1.18) are key cellular enzymes that catalyze the conjugation of glutathione with reactive intermediates, contributing to the cellular defence against oxidative stress in semen (Ketterer et al., 1983). The role of GST genes in determining semen quality (Olshan et al., 2010) and genetic susceptibility of infertility with impaired spermatogenesis in men (Xu et al., 2013; Ying et al., 2013) have been studied earlier. Several studies have pointed out the association between structural variations in

\footnotetext{
*Corresponding Author, E mail: dramukherjee@ wbuafscl.ac.in
} 
GSTs genes with oligoazoospermia, azoospermia and idiopathic male infertility risk (Aydemir et al., 2007; Wu et al., 2008; Finotti et al., 2009; Roshdy et al., 2014) or DNA damage of sperm exposed to air pollution (Rubes et al., 2007). In bulls also, GlutathioneS-transferase Mul isoform (GSTM1) is supposed to have a role in determining semen quality as a non-synonymous mutation has been associated with sperm motility and ATP content in frozen-thawed semen (Hering et al., 2015).

Progress in computer algorithms and tools has made it possible to explore the molecular structure of proteins and bigger molecular assemblies in depth (Chatron et al., 2017; Srivastava et al., 2018). Computational prediction of the $3 \mathrm{D}$ structure of a protein is useful in many areas of biomedicine, ranging from approximate family assignments to drug designing (Zhang, 2009). GSTM1, a key molecular regulator of bull semen quality, is a $25.8 \mathrm{kDa}$ protein present in the cytosol. We hypothesize that in silico prediction of GSTMI gene structure and function will add to the existing knowledge of underlying regulatory mechanism of bull sperm quality and fertility. Considering the importance of GSTM1 gene and its possible role as a candidate gene in bull semen quality, the present study has attempted to accurately predict the three-dimensional structure of GSTM1 protein in bovine.

\section{MATERIALS AND METHODS}

Retrieval and analysis of primary protein sequence: The primary protein sequence of bovine GSTM1 protein (Accession no: AAI34566.1) was retrieved from the NCBI database to use as a reference for homology search. ProtParam tool (http://web.expasy.org/ protparam/) was employed for the calculation of different physicochemical properties such as theoretical isoelectric point $(\mathrm{pI})$, molecular weight, the total number of positively and negatively charged residues, and instability and aliphatic indices of GSTM1 protein (Gasteiger et al., 2007). Protein domain families were searched from the Uniprot database (www.uniprot.org). The CELLO2GO web server (http://cello.life.nctu.edu.tw/cello2go/) was used for finding the probable subcellular localization and functional annotations of the predicted protein (Yu et al., 2014).

Three-dimensional structure prediction: The secondary structure of GSTM1 protein was predicted using Phyre2 (Kelley et al., 2015). The three-dimensional models were generated using Modeller 9.24, I-TASSER, Phyre 2, and Swiss Model. A suitable template, 1XW6 (Glutathione-S-Transferase of Homo sapiens), was identified for bovine GSTM1 based on maximum identity and query coverage using DELTA-BLAST tool (Boratyn et al., 2012). The Modeller 9.24 predicted ten prototypes of threedimensional structural forms using this template (Marti-Renom et al., 2000). Out of the generated models, the best model for GSTM1 was chosen based on the lowest DOPE (Discrete Optimized Protein Energy) score. I-TASSER (Iterative Threading ASSEmbly Refinement) is a hierarchical approach to protein structure and function prediction. It can construct full-length atomic models using iterative template-based fragment assembly simulations (Roy et al., 2010). SWISS-MODEL template library (SMTL) annotates biologically relevant ligand molecules, and a modeling machine uses this annotation to build the high-quality quaternary structure (Waterhouse et al., 2018). Protein structure prediction was done using Phyre 2, an advanced distant homology identification approach. This can predict ligand-binding sites and assess the impact of amino acid substitutions. (e.g., non-synonymous SNPs (nsSNPs)) for a given protein sequence (Kelley et al., 2015).

Model validation: The best models were predicted with this software, and the structures were refined using the GalaxyRefine server (http://galaxy.seoklab.org/cgi-bin/ submit.cgi?type=REFINE) (Heo et al., 2013). The stereochemical stability of the predicted 
model was further assessed using different protein quality based parameters such as the Ramachandran plot. The plot that evaluates the percentage residues lying in favored and allowed regions, the number of glycine and proline residues and orientation of dihedral angles including phi $(\Phi)$ and psi $(\psi)$ and backbone conformation was analyzed by PROCHECK module (https://servicesn.mbi. ucla.edu/PROCHECK/) of the PDBSum server (Laskowski et al., 2005), and also confirmed using the RAMPAGE server (Lovell et al., 2003). ProTSAV score (http://www.scfbioiitd.res.in/software/proteomics/protsav.jsp) i.e. a combined value of the quality assessment at various interfaces were determined. Identification of different regions in the protein structure with respect to aromatic receptor surface, hydrogen bonds, hydrophobic regions, regions for inter-polar charges, ionizability were executed using Discovery Studio (Biovia).

Molecular docking and docking complex visualization: The GSTM1 model protein was selected, and glutathione was chosen as a ligand from the drug bank database. The chosen ligand was docked using the PatchDock docking server (Mashiach et al., 2010). PatchDock is a geometry-based, rigid molecular docking algorithm that operates by object recognition and image segmentation techniques used in Computer Vision. The recommended clustering root-mean-square deviation (RMSD) of $4.0 \AA$ was used as the default setting for PatchDock analysis. The data of 500 docked complexes were chosen from the solution list. The poses generated were visualized with UCSF Chimera, Discovery Studio (Biovia) and receptor-ligand interaction was analyzed by Ligplot plus (Wallace et al., 1995).

Analysis of protein-protein interaction network: The STRING (Search Tool for the Retrieval of Interacting Proteins) analysis was carried out (https://string-db.org/) with a minimum required interaction score of 0.700 to find out the network of interaction with other proteins (Franceschini et al., 2013).

\section{RESULTS}

Retrieval and analysis of primary protein sequence: Bovine GSTM1 is a 218 amino acid residues long protein with $25.78 \mathrm{kDa}$ molecular weight and mainly demonstrated slightly acidic characteristics with 6.60 theoretical pI value (Table 1). CELLO2GO server confirmed that the protein is present in the cytoplasm, associated with the glutathione metabolism process and having transferase activity (84\%) (Fig. 1)

Three-dimensional structure prediction of GSTM1 and model validation: The threedimensional structure of GSTM1 protein was predicted by Modeller 9.24, I-TASSER, Phyre 2 and Swiss Model. From the input sequence and the given template molecule, Modeller 9.24 predicted a total of 10 structural prototypes. The individual DOPE score of each prototype was calculated by Modeller 9.24, and structure

Table 1. Physicochemical parameters of bovine GSTM1 protein

\begin{tabular}{ll}
\hline Physical and chemical parameters & GSTM1 \\
\hline Number of amino acids & 218 \\
Theoretical pI & 6.60 \\
Molecular weight & 25779.82 \\
Instability index & 37.01 \\
Aliphatic index & 81.93 \\
Total number of negatively charged residues (Asp + Glu) & 32 \\
Total number of positively charged residues (Arg + Lys) & 31 \\
Grand average of hydropathicity (GRAVY) & -0.416 \\
\hline
\end{tabular}


(Model 3, DOPE score: -28162.236328) having the lowest DOPE score was chosen. The best structures predicted by Modeller 9.24, ITASSER, Phyre 2, and Swiss Model were further refined by the GalaxyRefine server (Fig. 2). The Ramachandran Plot analysis (Table 2) revealed that the model predicted by Modeller 9.24 was the best model as it had a maximum of $99.54 \%$ residues [98.14\% in favored region $+1.4 \%$ in allowed region] remaining in favored region compared to $97 \%$ in favored region and $2.6 \%$ in the allowed region of the experimentally solved and X-ray resolved template structure (1XW6). ProTSAV score i.e. a combined value of the quality assessment at various interfaces, revealed that the predicted protein was stable and had RMSD values in the range of a good model (at the green-yellow interface) (Fig. 3). The RMSD deviation on superposition along the protein carbon backbone was $0.35 \AA$. Different regions in the GSTM1 protein structure with respect to aromatic receptor surface, hydrogen bonds, interpolated charges and hydrophobicity were calculated (Fig. 4).

Molecular docking: The model built using Modeller 9.24 was used for docking analysis using glutathione as a free ligand. The ligand was docked with the bovine GSTM1 model protein using PatchDock software (Fig. 5A). A Ligplot plus analysis of Glutathione (purple in colour) showing $\mathrm{H}$-bond and hydrophobic interactions with the bovine GSTM1 are demonstrated in Fig. 5B and 5C. Ligplot plus analysis shows hydrogen bonding inter-actions between the hydrogen atom of the $-\mathrm{OH}$ group of the active site amino acid residues Thr186 and $-\mathrm{COOH}$ group of Glu189 in this docked complex. PatchDock was used to create many docked structures for the GSTM1-Glutathione complex, which were ranked based on geometric shape complementary score, interface area, and atomic contact energy. The highest-ranked structure had a geometric shape complementarity score of 3602 , an approximate interface area of $410.50 \AA$ and atomic contact energy (ACE) of the approximate interface area of $-47.83 \mathrm{kcal} / \mathrm{mol}$ as shown in Fig. 5A. Further, the generated results were refined using the FireDock server.

Protein-protein interaction: Protein-protein interaction study done by STRING analysis showed 10 probable interacting partners of bovine GSTM1 viz. GSTA4 (Gltathione-STransferase A4), GSTM2 (Gltathione-STransferase M2), GSTM3 (Gltathione-STransferase M3), GSTO1 (Gltathione-STransferase O1), GSTO2 (Gltathione-STransferase O2), GSTT1 (Gltathione-STransferase T1), GSTT3 (Gltathione-STransferase T3), CYP1A1 (Cytochrome P450 1A1), CYP2E1 (Cytochrome P450 2E1) and LOC785216 (Fig. 6).

\section{DISCUSSION}

Three-dimensional structure prediction of GSTM1 protein and its validation: Ten best structures were predicted by Modeller 9.24 along with DOPE score of each prototype. Graph of DOPE score was obtained for ten possible structures and the structure with more negative value was considered to be the best. The possible structures were predicted by ITASSER, Phyre 2 and SWISS-MODEL also (Fig. 2). All these models were further validated by analyzing the Ramachandran plot (Table 2).

Table 2. Evaluation of residues in Ramachandran Plot as prepared from RAMPAGE

\begin{tabular}{|c|c|c|c|}
\hline Protein structure & Favored region(\%) & Allowed region(\%) & Outlier region (\%) \\
\hline MODELLER & 98.14 & 1.4 & 0.5 \\
\hline I-TASSER & 97.7 & 1.4 & 0.9 \\
\hline Phyre2 & 96.3 & 2.8 & 0.9 \\
\hline SWISS-MODEL & 96.3 & 2.8 & 0.9 \\
\hline
\end{tabular}


Indian Journal of Animal Health, December, 2021

Computational insight into bovine GSTM1 gene
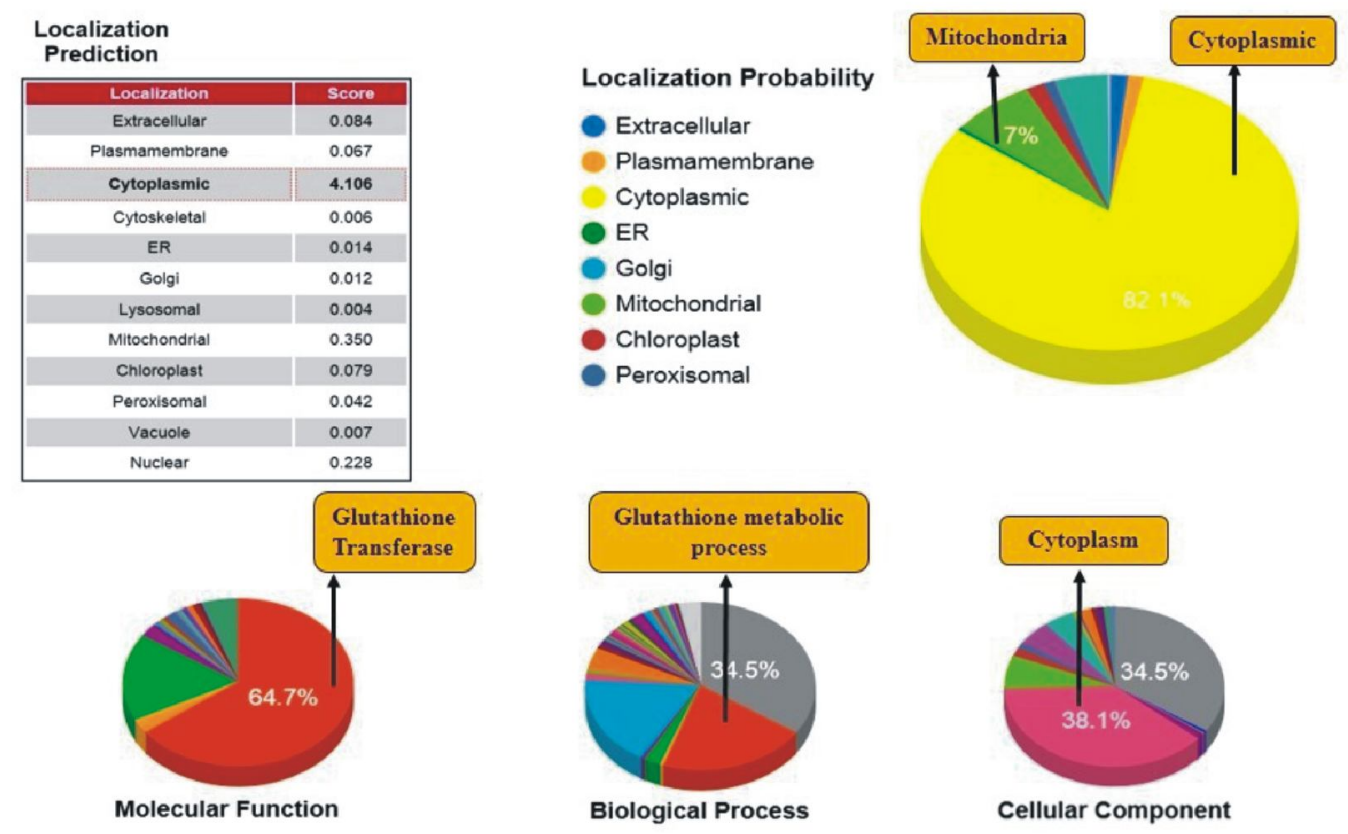

Fig. 1. Functional annotation of the bovine GSTM1 protein was measured in the form of gene ontology enrichment analysis. The three ontological terms used were the molecular function, biological process involved, and cellular location. The subcellular localization of the protein was shown as a big pie chart and was retrieved by CELLO2GO webserver.

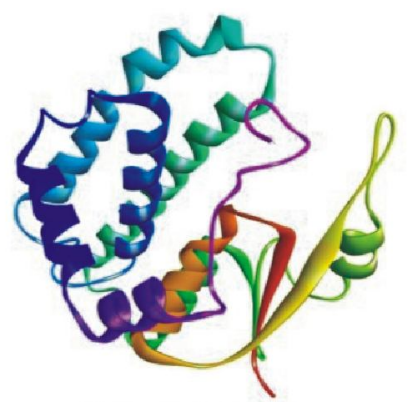

MODELLER 9.24

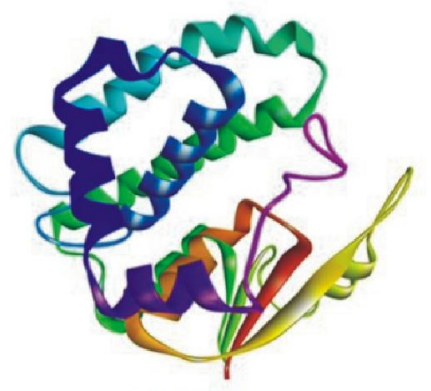

PHYRE2

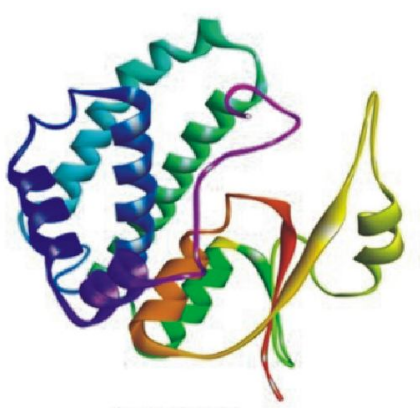

I-TASSER

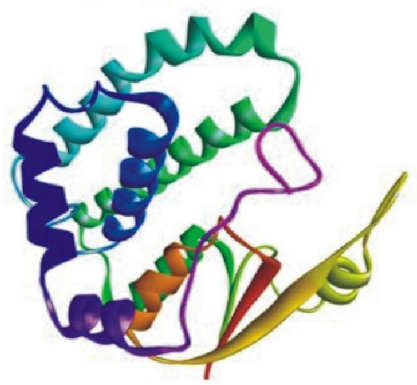

SWISS MODEL

Fig. 2. Predicted three dimensional structures of bovine GSTM1 after refinement by Galaxy Refine server. Predictions were done by Modeller9.24, I-TASSER, Phyre2 and Swiss-Model. The structure predicted by Modeller 9.24 was the best structure based on Ramachandran Plot analysis and other methods of validation. 
Indian Journal of Animal Health, December, 2021

Computational insight into bovine GSTM1 gene

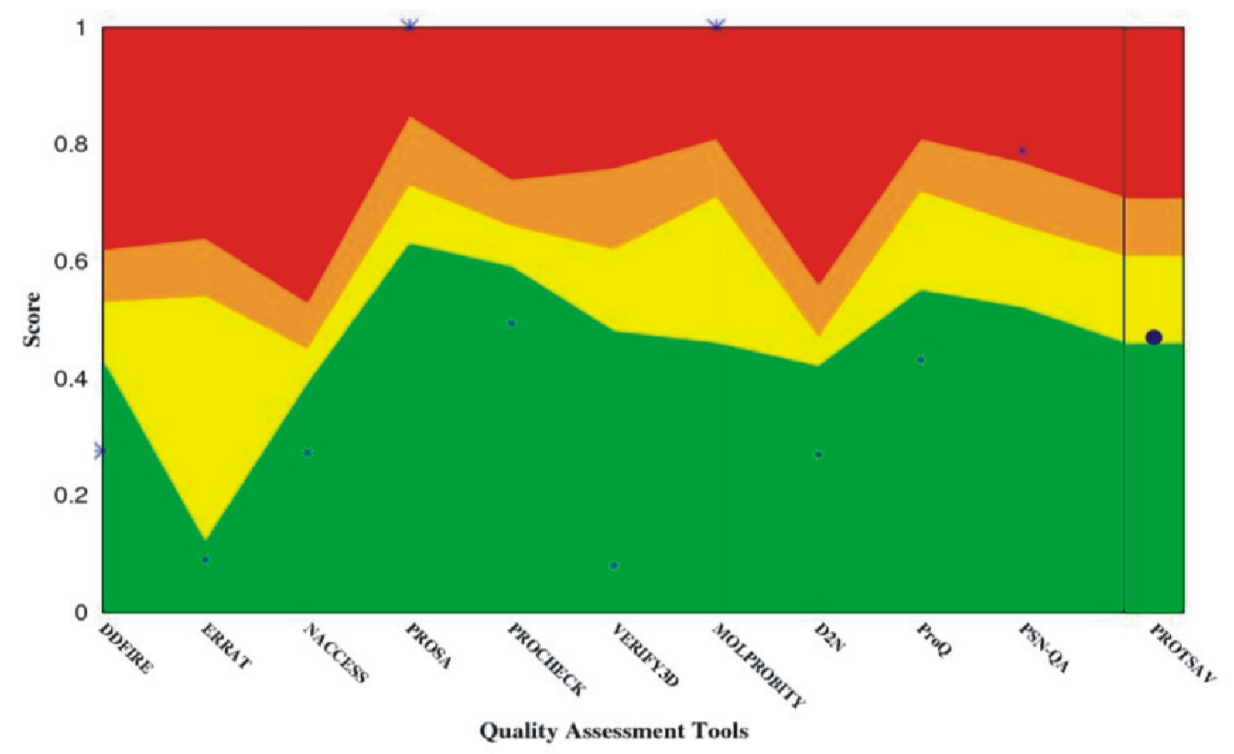

Fig. 3. The qualitative assessment of the modelled protein (bovine GSTM1) based on ProTSAV score. The ProTSAV assessed the predicted model structures, based on some popular online servers and standalone tools, and furnishes with a single quality score in case of individual protein structure, along with a graphical representation and ranking in case of multiple protein structure assessment. In our results, the ProTSAV score was found close to green-yellow interface signifying that the model has reasonable stability and accuracy in terms of qualitative and quantitative parameters.
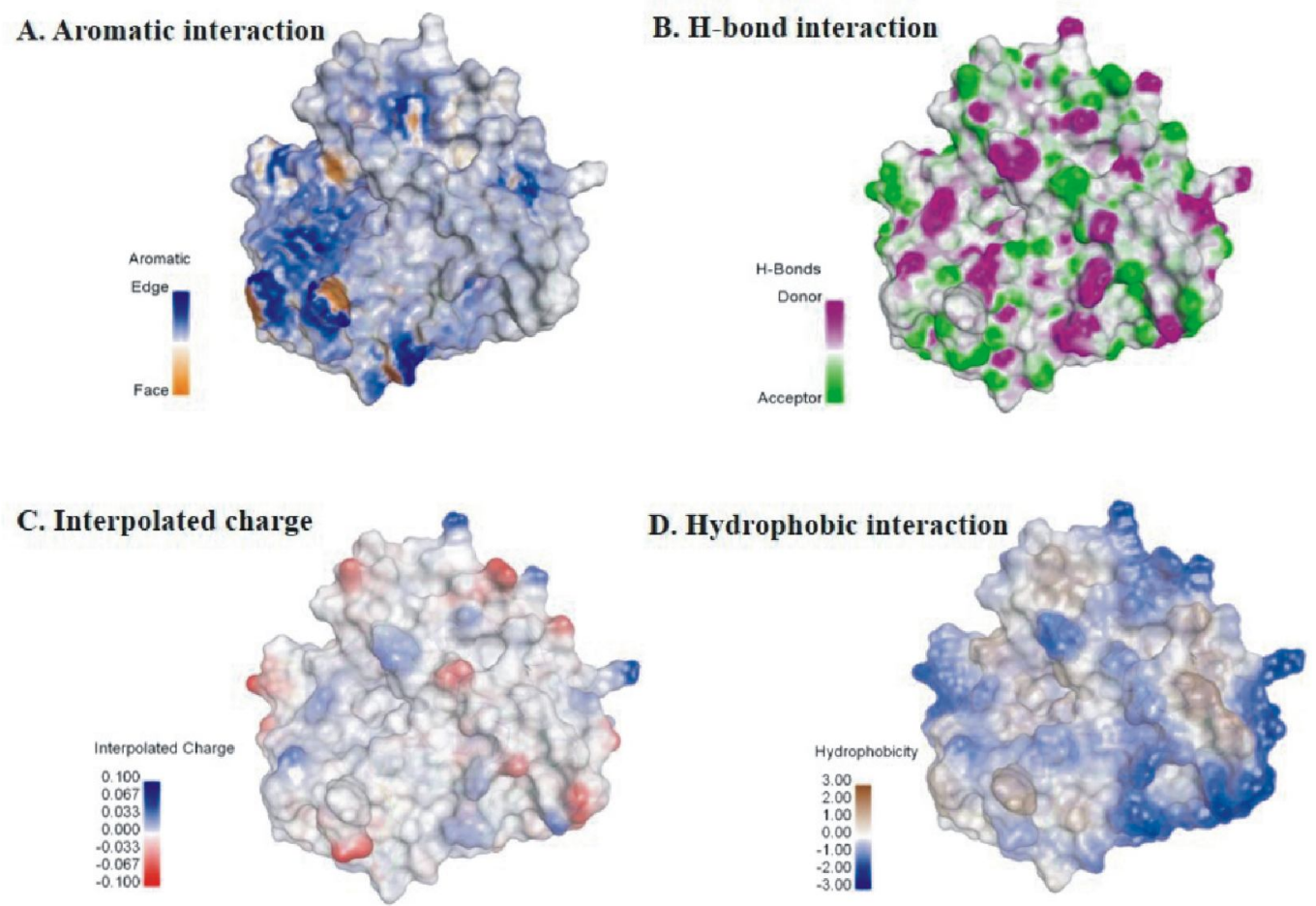

Fig. 4. Different regions in the GSTM1 protein structure with respect to (A) Aromatic Receptor Surface, (B) Hydrogen Bonds, (C) Interpolated charges and (D) Hydrophobic interaction 
Indian Journal of Animal Health, December, 2021

Computational insight into bovine GSTM1 gene

\section{A. GSTM1-ligand interaction}

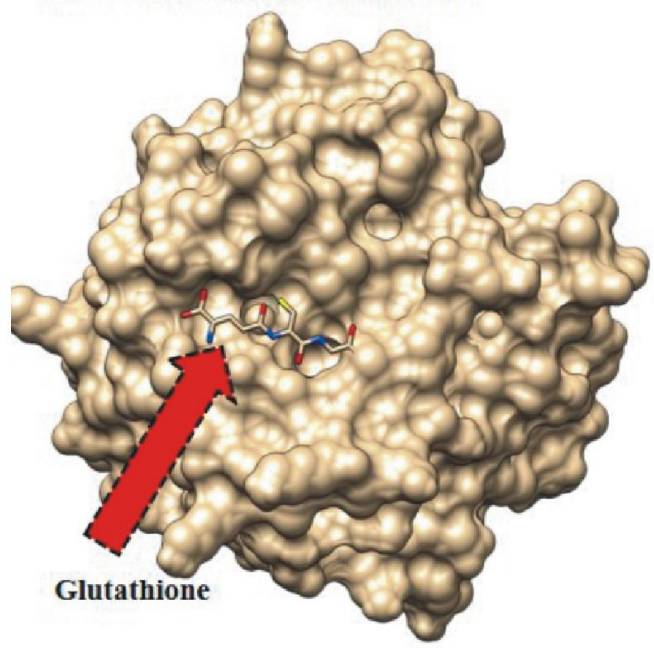

B. H-bond interaction

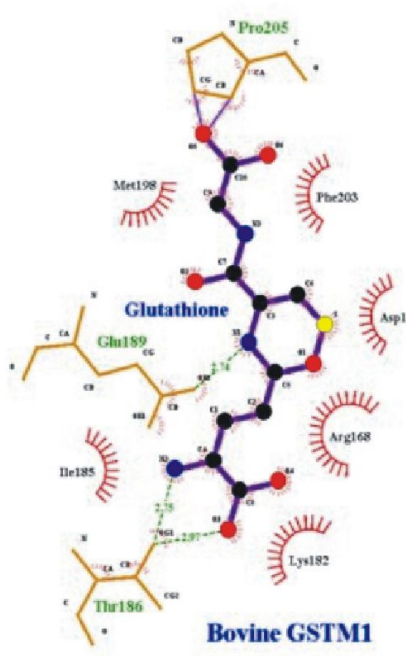

C. Hydrophobic interaction

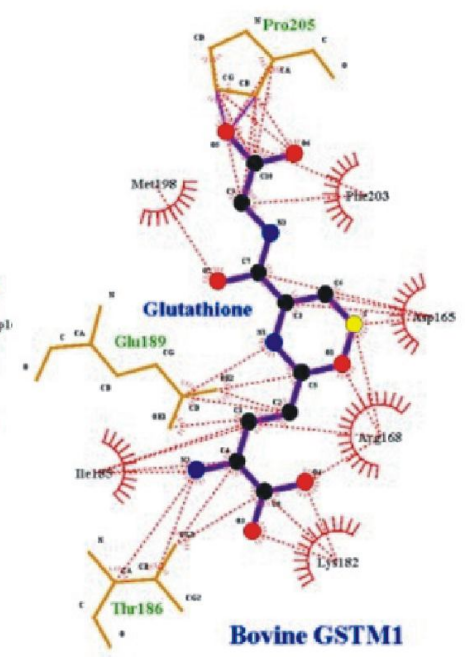

Fig. 5. (A) Prediction of bovine GSTM1-ligand interaction by PatchDock and FireDock server. This is the highest ranked structure based on their geometric shape complementary score, interface area, and atomic contact energy. (B) Ligplot plus analysis shows hydrogen bonding interactions between the hydrogen atom of the -OH group of the active site amino acid residues Thr186 and COOH group of Glu189 in this docked complex. (C) Ligplot plus analysis shows hydrophobic interactions formed between the ligand and Asp165, Arg168, Lys182, Ile185, Thr186, Glu189, Met198, Phe203 and Pro205.

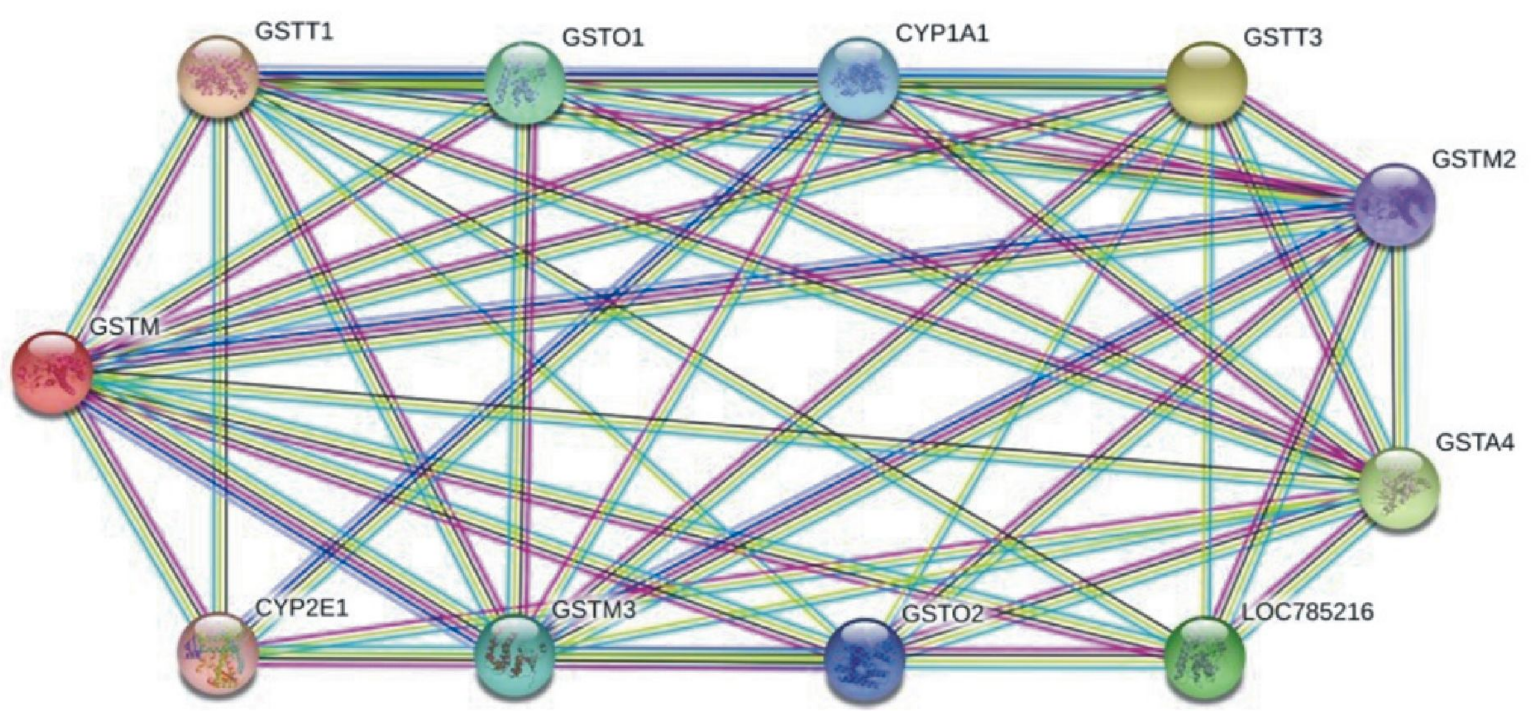

Fig. 6. Interacting partners of bovine GSTM1 protein revealed by STRING analysis 
Ramachandran plot assesses $\Phi$ and $\psi$ backbone torsion angles of each residue in a protein. The more the number of amino acid residues in the favored regions, the protein structure will be more stable (Lovell et al., 2003). Ramachandran Plot analysis by RAMPAGE server grouped the amino acid residues, and maximum stability was observed in protein structure predicted by Modeller 9.24 (Table 2). Considering all the structures predicted by different structure prediction tools, the structure predicted by Modeller 9.24 was selected as the most acceptable model of bovine GSTM1 protein. This was also confirmed from the ProtSAV score values (Fig. 3) as all the qualitative parameters measured, the predicted model lying in the zone of good resolution (2.05.0Å). ProTSAV is a meta-server with a set of model quality evaluation algorithms. It analyses a protein's quality and accuracy of the protein structural model. It calculates a global quality score for the input structure that has been supplied. This global quality score is generated from the user's judgment of the quality of several validation tools (modules).

Intra-protein characterization: Intra-protein characterization of a protein model structure is necessary to study diverse aspects of the protein function. Interactions between the aromatic amino acid residues have a major impact on the protein structures. Although these interactions individually contribute little to the conformational stability of the protein structure, their combined action act as a major determinant of the overall structure and conformation of the protein molecules (Anjana et al., 2012). Fig. 4A depicts several colour representations of aromatic residues found on the edges and faces, as well as at intermediate places. Hydrogen bonds (H-bonds) confer rigidity to the protein structure and provide most of the directional interactions that underpin protein folding, protein structure and molecular recognition (Sheu et al., 2003). Different H-bonds donor and acceptor sites in the bovine GSTM1 protein structure underscores stability imparted by H-bonds to the protein structure (Fig. 4B). Charges are considered an integral part of protein structure and function, enhancing solubility and providing specificity in molecular interactions (Højgaard et al., 2016). Interpolated charges in the modelled protein structure ranging from -1 to +1 with different colour configurations have been depicted in Fig. 4C. Hydrophobic interactions are a major driving force in protein folding and stability (Camilloni et al., 2016). Fig. 4D represents the hydrophobic surface of the protein structure.

Molecular Docking: In this study, online protein-ligand interaction tools PatchDock and FireDock server were used as docking tools for glutathione with bovine GSTM1 protein. Glutathione as a ligand was docked against the 3D structure of GSTM1 protein (Fig. 5A). The docking run was done using the PatchDock server, and all the results generated by this server showed a very high binding affinity score. Furthermore, the generated results were refined using the FireDock server, and all the results strongly support the PatchDock score with excellent binding energy.

For the effective binding of a ligand to a protein receptor, the ligand's affinity for the protein and the stability of the protein-ligand complex are two critical requirements. The previously mentioned atomic contact energy (ACE), along with the interaction profile (hydrogen bonds and hydrophobic interactions), ensure the maximum affinity and stability of ligand with the protein. To gain insight on the binding modes of glutathione as per the molecular docking results, H-bond interactions and hydrophobic interactions were analyzed and visualized by Ligplot plus analysis (Fig. 5B and 5C). Interaction profiles were figured out only for the best possible orientation of the ligand molecule. For LigPlot plus analysis, an illustrative figure was generated, describing bonded as well as non-bonded interactions between the ligands and protein.

Protein-protein interaction: Fig. 6 shows an interactomic map of GSTM1 protein in bovine. STRING analysis identified ten functional 
partners with a high confidence score (0.700). The list of identified interaction partners and their probable functional roles are summarized below:

GSTT1- Glutathione S-transferase, theta 1, conjugation of reduced glutathione to a wide number of exogenous and endogenous hydrophobic electrophiles. It also binds steroids, bilirubin, carcinogens and numerous organic anions.

GSTT3- Glutathione S-transferase, theta 3 GSTA4- Conjugation of reduced glutathione to a wide number of exogenous and endogenous hydrophobic electrophiles

LOC785216- Uncharacterized protein

GSTO1- Glutathione S-transferase omega 1

GSTM3 - Glutathione S-transferase mu 3

CYP1A1- Cytochromes P450 is a group of heme-thiolatemonooxygenases

GSTO2 - Glutathione S-transferase omega 2

CYP2E1- Metabolizes several precarcinogens, drugs, and solvents to reactive metabolites; belongs to the cytochrome P450 family.

One of the factors limiting the implementation of genomic selection in the context of Indian dairy sector is wide genomic variations among diverse breeds of cattle. Structural variations of the genes governing the key economic traits like fertility are of immense

\section{REFERENCES}

Anjana R, Vaishnavi MK, Sherlin D, Kumar SP, Naveen $\mathrm{K}$ et al., 2012. Aromatic-aromatic interactions in structures of proteins and protein-DNA complexes: A study based on orientation and distance. Bioinformation, 8(24): 1220-1224, doi: $10.6026 / 97320630081220$

Aydemir B, Onaran I, Kiziler AR, Alici B and Akyolcu MC, 2007. Increased oxidative damage of sperm and seminal plasma in men with idiopathic infertility is higher in patients with glutathione S-transferase Mu-1 null genotype. Asian J Androl, 9(1): 108-115, doi: 10.1111/j.1745-7262. 2007.00237.x importance to select the animals for optimum production. Theoretical knowledge about the structure and function of key proteins is essential to understand the impact of structural genomic variations on protein function. Based on the present study, future research attempts can be made to investigate how the SNPs in GSTM1 gene can alter the structure of the corresponding protein, which, in turn, will make the approach of bull selection more pragmatic. In the present scenario, there is a need for a rigorous selection of bulls based on genomic variants along with the in silico gene analysis technique to get optimum conception rate from artificial insemination program in the dairy industry, thereby helping the farmers to get the maximum benefit from rearing cattle. GSTM1 is a gene of importance in this context as it has a special role in determining sperm motility and ATP content in a bull. However, our knowledge about the structural and functional roles of GSTM1 gene and protein in bovine is scanty. Therefore, characterization of the bovine GSTM1 gene and structural prediction of its protein equivalent will provide useful information for better understanding its function in determining the quality of bull sperm. Also, the study will be a significant theoretical resource to conduct future studies aiming to develop candidate gene markers for selecting superior quality bulls.

Conflict of interest: Authors have no conflict of interest in this study.

Boratyn GM, Schäffer AA, Agarwala R, Altschul SF, Lipman DJ et al., 2012. Domain enhanced lookup time accelerated BLAST. Biol Direct, 7(1): 12, doi: 10.1186/1745-6150-7-12

Byrne K, Leahy T, McCulloch R, Colgrave ML and Holland MK, 2012. Comprehensive mapping of the bull sperm surface proteome. Proteomics, 12(23-24): 3559-3579, doi: 10.1002/pmic. 201200133

Camilloni C, Bonetti D, Morrone A, Giri R, Dobson CM et al., 2016. Towards a structural biology of the hydrophobic effect in protein folding. Sci Rep, 6: 28285, doi: 10.1038/srep28285 


\section{Computational insight into bovine GSTM1 gene}

Chatron N, Chalmond B, Trouvé A, Benoît E, Caruel H et al., 2017. Identification of the functional states of human vitamin $\mathrm{K}$ epoxide reductase from molecular dynamics simulations. RSC Adv, 7(82): 52071-52090, doi: 10.1039/C7RA07463H

Finotti ACF, Costa E Silva RCP, Bordin BM, Silva CTX and Moura KKVO, 2009. Glutathione Stransferase M1 and T1 polymorphism in men with idiopathic infertility. Genet Mol Res, 8(3): 10931098, doi: 10.4238/vol8-3gmr642

Franceschini A, Szklarczyk D, Frankild S, Kuhn M, Simonovic M et al., 2013. STRING v9.1: proteinprotein interaction networks, with increased coverage and integration. Nucleic Acids Res, 41: D808-D815, doi: 10.1093/nar/gks1094

García-Ruiz A, Cole JB, VanRaden PM, Wiggans GR, Ruiz-López FJ et al., 2016. Changes in genetic selection differentials and generation intervals in US Holstein dairy cattle as a result of genomic selection. Proc Nat Acad Sci, 113(28): E3995E4004, doi: 10.1073/pnas.1519061113

Gasteiger E, Hoogland C, Gattike A, Duvaud S, Wilkins MR et al., 2007. A protein identification and analysis tools on the ExPASy server, Humana, Louisville, pp 571-607

Heo L, Park H and Seok C, 2013. GalaxyRefine: protein structure refinement driven by side-chain repacking. Nucleic Acids Res, 41(W1): W384W388, doi: 10.1093/nar/gkt458

Hering DM, Lecewicz M, Kordan W, Majewska A and Kaminski S, 2015. Missense mutation in glutathione-S-transferase M1 gene is associated with sperm motility and ATP content in frozenthawed semen of Holstein-Friesian bulls. Anim Reprod Sci, 159: 94-97, doi: 10.1016/ j.anireprosci.2015.06.001

Højgaard C, Kofoed C, Espersen R, Johansson KE, Villa $\mathrm{M}$ et al., 2016. A soluble, folded protein without charged amino acid residues. Biochemistry, 55: 3949-3956, doi: 10.1021/acs.biochem.6b00269

Jodar M, Sendler E, Moskovtsev SI, Librach CL, Goodrich R et al., 2015. Absence of sperm RNA elements correlates with idiopathic male infertility. Sci Transl Med, 7: 295re6, doi: 10.1126/scitranslmed.aab1287

Kelley LA, Mezulis S, Yates CM, Wass MN and SJE, 2015. The Phyre2 webportal for protein modeling, prediction and analysis. Nat Protoc, 10: 845-858, doi: $10.1038 /$ nprot.2015.053

Ketterer B, Coles B and Meyer DJ, 1983. The role of glutatione in detoxification. Environ Health Perspect, 49: 59-69, doi: 10.1289/ehp.834959
Laskowski RA, Chistyakov VV and Thornton JM, 2005. PDBsum more: new summaries and analyses of the known 3D structures of proteins and nucleic acids. Nucleic Acids Res, 33(sppl_1): D266-D268, doi: 10.1093/nar/gki001

Lovell SC, Davis IW and Arendall PIW, 2003. Structure validation by Calpha geometry: phi, psi and Cbeta deviation. Proteins, 50: 437-450, doi: 10.1002/ prot. 10286

Marti-Renom MA, Stuart A, Fiser A, Sánchez R, Melo F et al., 2000. Comparative protein structure modeling of genes and genomes. Annu Rev Biophys Biomol Struct, 29: 291-325, doi: 10.1146/annurev.biophys.29.1.291

Mashiach E, Schneidman-Duhovny D, Peri A, Shavit Y, Nussinov R et al., 2010. An integrated suite of fast docking algorithms. Proteins, 78(15): 31973204, doi: $10.1002 /$ prot. 22790

Olshan AF, Luben TJ, Hanley NM, Perreault SD, Chan RL et al., 2010. Preliminary examination of polymorphisms of GSTM1 GSTT1, and GSTZ1 in relation to semen quality. Mutat Res, 688(12): 41-46, doi: 10.1016/j.mrfmmm.2010.03.002

Roshdy OH, Hussein TM, Zakaria NH and Sabry AA, 2014. Glutathione S-transferase $\mathrm{Mu}-1$ gene polymorphism in Egyptian patients with idiopathic male infertility. Andrologia, 47(5): 587-593, doi: 10.1111/and.12306

Roy A, Kucukural A and Zhang Y, 2010. I-TASSER: A unified platform for automated protein structure and function prediction. Nat Protoc, 5: 725-738, doi: $10.1038 /$ nprot.2010.5

Rubes J, Selevan SG, Sram RJ, Evenson DP and Perreault SD, 2007. GSTM1 genotype influences the susceptibility of men to sperm DNA damage associated with exposure to air pollution. Mutat Res, 625(1-2): 20-28, doi: 10.1016/j.mrfmmm. 2007.05.012

Selvaraju S, Parthipan S, Somashekar L, Kolte AP, Binsila BK et al., 2017. Occurrence and functional significance of the transcriptome in bovine (Bos taurus) spermatozoa. Sci Rep, 7(1): 42392, doi: $10.1038 /$ srep42392

Sheu SY, Yang DY, Selzle HL and Schlag EW, 2003. Energetics of hydrogen bonds in peptides. Proc Nat Acad Sci USA, 100(22): 12683-12687, doi: 10.1073/pnas.2133366100

Srivastava A, Nagai T, Srivastava A, Miyashita O and Tama F, 2018. Role of computational methods in going beyond X-ray crystallography to explore protein structure and dynamics. Int $\mathbf{J}$ Mol Sci, 19(11): 3401, doi: 10.3390/ijms 19113401 
Wallace AC, Laskowski RA and Thornton JM, 1995. LigPlot: A program to generate schematic diagrams of protein-ligand interactions. Protein Eng Des Sel, 8(2): 127-134, doi: 10.1093/protein/ 8.2.127

Waterhouse A, Bertoni M, Bienert S, Studer G, Tauriello G et al., 2018. SWISS-MODEL: homology modelling of protein structures and complexes. Nucleic Acids Res, 46(Supply.1): W296-W303, doi: $10.1093 /$ nar/gky427

Wu QF, Xing JP, Tang KF, Xue W, Liu M et al., 2008. Genetic polymorphism of glutathione Stransferase T1 gene and susceptibility to idiopathic azoospermia or oligospermia in north western China. Asian J Androl, 10(2): 266-270, doi: 10.1111/j.1745-7262.2008.00347.x

Xu XB, Liu SR, Ying HQ and Zhou CA, 2013. Null genotype of GSTM1 and GSTT1 may contribute to susceptibility to male infertility with impaired spermatogenesis in Chinese population. Biomarkers, 18(2): 151-154, doi: 10.3109/ 1354750X.2012.755221

Ying HQ, Qi Y, Pu XY, Liu SR and Cun AZ, 2013. Association of GSTM1 and GSTT1 genes with the susceptibility to male infertility: result from a meta-analysis. Genet Test Mol Biomark, 17(7): 535-542, doi: 10.1089/gtmb.2012.0409

Yu CS, Cheng CW, Su WC, Chang KC, Huang SW et al., 2014. CELLO2GO: A Web Server for Protein subCELlular LOcalization prediction with functional gene ontology annotation. PLoS One, 9(6): e99368, doi: 10.1371/journal.pone. 0099368

Zhang Y, 2009. Protein structure prediction: is it useful? Curr Opin Struct Biol, 19(2): 145-155, doi: 10.1016/j.sbi.2009.02.005

Received 14.07.2021, Accepted - 04.11.2021, Published - 19.11.2021 (Online), 01.12.2921 (Print)

Section Editor: Dr. I. Samanta, Associate Editor 